\title{
Characterization and Noise Analysis of Capacitive MEMS Acoustic Emission Transducers
}

\author{
Wei $\mathrm{Wu}$ and David W. Greve \\ Department of Electrical and Computer Engineering \\ Carnegie Mellon University \\ Pittsburgh, PA 15213 \\ wwu@cmu.edu
}

\author{
Irving J. Oppenheim \\ Department of Civil and Environmental Engineering \\ Carnegie Mellon University \\ Pittsburgh, PA 15213
}

\begin{abstract}
Resonant type capacitive MEMS transducers were fabricated using a Multi-User MEMS Process (MUMPs) for the detection of acoustic emission (AE). Electrical and mechanical characterization of the MEMS transducers has been performed. The performance of the transducers is limited by the noise. In this paper, we present the noise analysis, the discussion of noise sources, and show that Brownian noise plays a significant role in the capacitive MEMS transducers.
\end{abstract}

\section{INTRODUCTION}

Acoustic emission is an ultrasonic wave generated when elastic energy is released in a structure by permanent and irreversible change. As a nondestructive evaluation method, acoustic emission testing is widely used to detect and locate faults in structures. Detection of acoustic emission events allows determination of stress conditions that have the potential to cause permanent damage. The frequency spectrum of acoustic emission events is between $100 \mathrm{kHz}$ and $1 \mathrm{MHz}$.

We report here a MEMS sensor that replaces conventional piezo-electric transducers for acoustic emission. In our design, a polysilicon plate is suspended by springs and set into motion when acoustic energy is coupled to the substrate. This results in a resonant-type transducer that is only sensitive to the Fourier components of the acoustic emission waveform near its resonant frequency. The sensitivity of such a transducer depends on the damping or quality factor of the resonator and in addition is limited by inherent Brownian noise.

In this paper we report on the construction and characterization of a MEMS acoustic emission transducer. In particular we will focus on the factors that determine the resonator damping and noise.

\section{TRANSDUCER DESIGN}

Fig. 1 shows the concept for an acoustic emission transducer. An ultrasonic wave produced by crack propagation is coupled to the bottom plate of a capacitive transducer. The top plate then vibrates with respect to the bottom plate, resulting in a time-varying voltage $v_{m}(t)$. This time-varying voltage is acquired and analyzed using a data acquisition system.

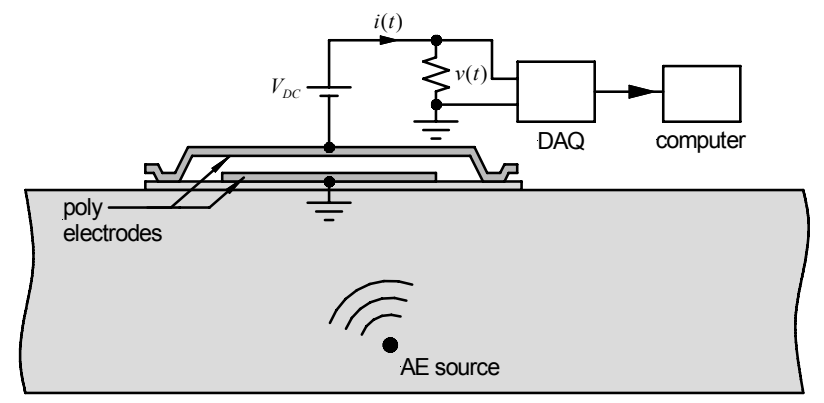

Figure 1. Concept for an acoustic emission transducer.

These transducers exhibit resonant behavior where the resonant frequency

$$
\omega_{0}=\sqrt{\frac{k}{m}}
$$

where $m$ is the effective mass of the top plate and $k$ is the spring constant of the supports. For such a transducer it can be shown that the maximum signal current $i_{m}$ resulting from a displacement of the bottom plate of amplitude $u_{m}$ is given by [1]

$$
i_{m}=V_{D C} \frac{\varepsilon_{0} A}{g^{2}} \omega_{0} Q u_{m}
$$

where $\varepsilon_{0}$ is the dielectric permittivity of vacuum, $g$ is the gap between the top and bottom plates of the capacitor, $A$ is the transducer area, $\omega_{0}$ is the resonant frequency, and $Q$ is the quality factor. The signal increases with the quality factor and as a result it is desirable to design transducers for a high $Q$ value. In transducers operated at atmospheric pressure damping is caused by both the radiation acoustic energy into air and squeeze-film damping associated with the movement of air into and out of the etch release holes. The design of 
the diaphragm shape and etch hole spacing determines the $Q$ of the transducer.

Fig. 2 illustrates the transducer design used in this work. The transducer is designed in the poly-MUMPS process [2] and uses POLY0 as the bottom electrode and POLY1 as the top electrode. The nominal gap between the electrodes is $1.25 \mu \mathrm{m}$. In this design the damping is reduced by close spacing of the etch release holes $(3.5 \mu \mathrm{m}$ square and arranged in a triangular array). Four transducers with different resonant frequencies were fabricated on a single $0.5 \mathrm{~cm} \times 0.5 \mathrm{~cm}$ chip. The resonant frequencies were obtained by varying the spring constant of the supports [3].
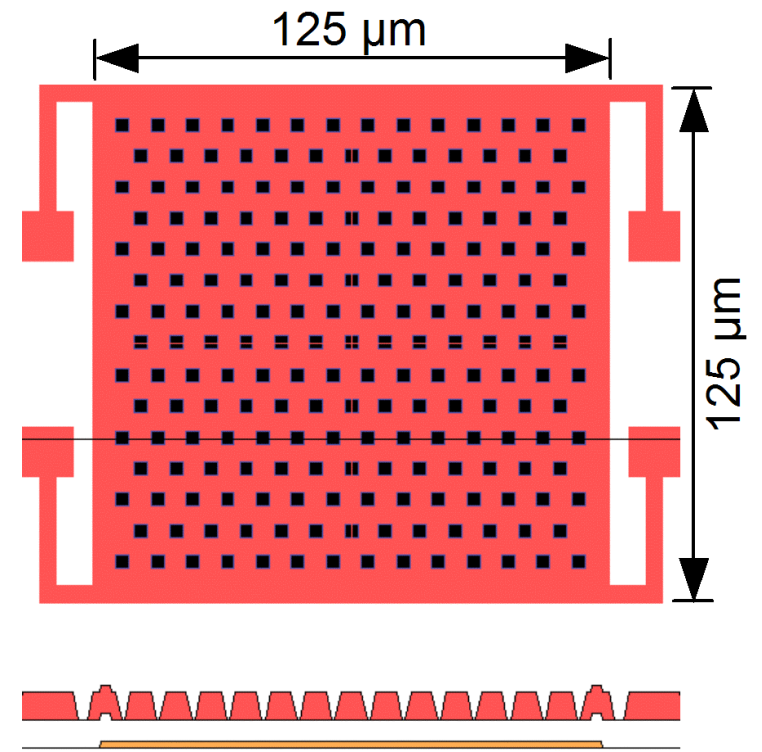

Figure 2. The top view and cross section of a $125 \mu \mathrm{m}$ square transducer unit with identical springs at each corner. The anchors are not shown..

\section{PACKAGING AND CHARACTERIZATION}

Completed chips were attached onto a 68 pin cavitydown ceramic package [4] using silver epoxy [5] and then wire-bonded. The inverted configuration of these packages gives a large flat surface suitable for attachment to the structure of interest.

Electrical characterization was performed in order to determine the resonant frequencies and $\mathrm{Q}$ of the completed transducers. Impedance measurements were performed using a HP 4192A LF impedance analyzer. Figure 3 shows the results of this measurement for one of the transducers obtained with a DC bias voltage of $12 \mathrm{~V}$. Characterization results for all transducer types are presented in Table I. These results show a moderate improvement in quality factor over a previous design with less optimum etch hole spacing [6].

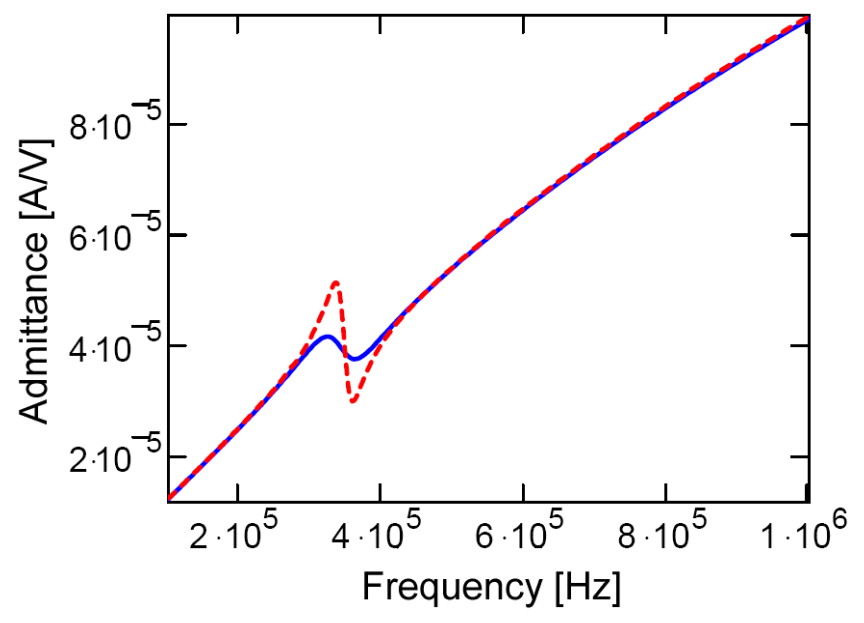

Figure 3. Measured admittance as a function of frequency for a $345 \mathrm{kHz}$ transducer: (solid line) data taken at atmospheric pressure showing a $Q$ of 5.4; and (dashed line) data from transducer in vacuum-sealed package.

Table I. Structural parameters of five different transducers and measured transducer parameters. $Q_{a t m}$ and $Q_{\text {sealed }}$ were measured at atmospheric pressure and in a sealed, evacuated package, respectively.

\begin{tabular}{|c|c|c|c|c|c|}
\hline $\begin{array}{c}\text { spring } \\
\text { length } \\
\boldsymbol{L}_{\boldsymbol{s}}[\boldsymbol{\mu \mathrm { m } ]}\end{array}$ & $\begin{array}{c}\text { spring } \\
\text { number } \\
\boldsymbol{n}_{\boldsymbol{s}}\end{array}$ & $\begin{array}{c}\boldsymbol{f}_{\text {s1 }}[\mathrm{kHz}] \\
(\text { simulated) }\end{array}$ & $\begin{array}{c}\boldsymbol{f}_{\text {s1 }}[\mathrm{kHz}] \\
(\mathbf{e x p t})\end{array}$ & $\begin{array}{c}\boldsymbol{Q}_{\text {atm }} \\
(\mathbf{e x p t})\end{array}$ & $\begin{array}{c}\boldsymbol{Q}_{\text {sealed }} \\
(\mathbf{e x p t})\end{array}$ \\
\hline 28.5 & 4 & 255 & 232 & 3.5 & 11.7 \\
\hline 17.5 & 4 & 351 & 311 & 4.8 & 13.8 \\
\hline 10.0 & 4 & 404 & 345 & 5.4 & 14.4 \\
\hline 10.5 & 8 & 500 & 443 & 5.5 & 19.7 \\
\hline
\end{tabular}

Packaged transducers have been integrated with amplifier and bias circuitry in order to obtain a compact unit that can be easily used in testing applications. Fig. 4 shows the completed transducer unit (without cover). The electronics is on two custom-design circuit boards and consists of four independent channels of amplification (gain of $100 \mathrm{~V} / \mathrm{V}$ ). Also included is bias circuitry including bypass capacitors to eliminate noise introduced through the bias channel. This unit connects directly to a National Instruments PCI-6110 DAQ board which simultaneously acquires signals from all four channels.

Integration of the amplifier, bias circuitry, and transducers results in a considerable reduction of electrically coupled noise, which in previous work has been a serious limitation [1]. Performance is now limited by the intrinsic noise of the transducers themselves. In the following section we discuss the magnitude of this noise and also possible options for further reduction in noise. 


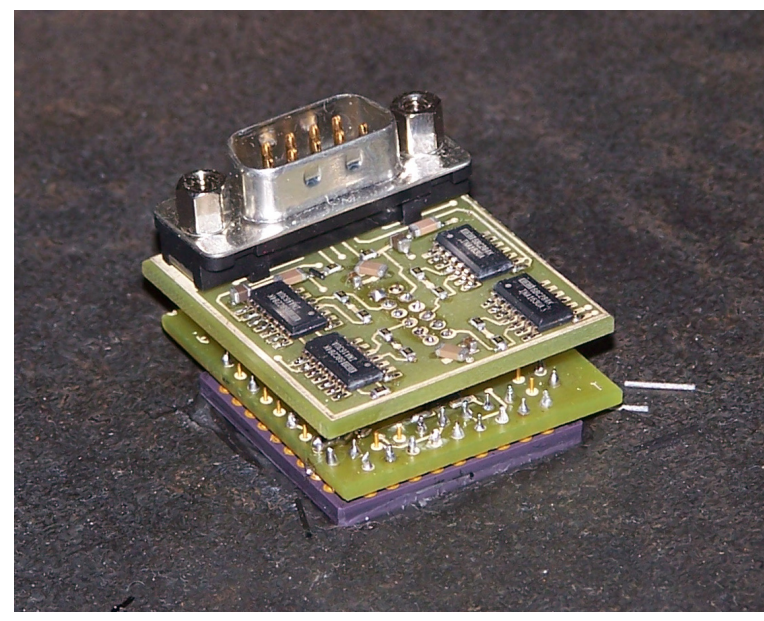

Figure 4. MEMS chip (four transducers) and printed circuit board mounted on a steel plate.

\section{TRANSDUCER NOISE}

The ultimate limit on transducer performance is determined by Brownian noise, caused by collisions between air molecules and the suspended diaphragm. The spectral density of the squared magnitude of the force $\left[\mathrm{N}^{2} / \mathrm{Hz}\right]$ caused by air molecules is independent of frequency and is given by [7]

$$
\left\langle\left|F_{B}(\omega)\right|^{2}\right\rangle=4 k_{B} T m \omega_{0} / Q
$$

where $k_{B}$ is the Boltzmann constant, $T$ the absolute temperature, $m$ the mass of the diaphragm and $\omega_{0} / Q$ is the mechanical damping coefficient. The equation of motion for the displacement $x(t)$ of the diaphragm is

$$
\ddot{x}+\frac{\omega_{0}}{Q} \dot{x}+\omega_{0}^{2} x=\frac{F_{B}(t)}{m},
$$

where $F_{B}(t)$ is the Brownian force. Taking the Fourier transform and solving for the velocity spectral density $V(\omega)$ $=j \omega X(\omega)$ gives

$$
V(\omega)=\frac{F_{B}(\omega) / m}{j \omega+\frac{\omega_{0}}{Q}+\frac{\omega_{0}^{2}}{j \omega}}
$$

from which we find for the ensemble average

$$
\left\langle|V(\omega)|^{2}\right\rangle=\frac{\left\langle\left|F_{B}(\omega) / m\right|^{2}\right\rangle}{\left(\omega-\frac{\omega_{0}^{2}}{\omega}\right)^{2}+\left(\frac{\omega_{0}}{Q}\right)^{2}} .
$$

The current in the external circuit is related to velocity spectral density through

$$
i(t)=\frac{d\left(C V_{D C}\right)}{d t}=V_{D C} \frac{d C}{d t}=\frac{V_{D C} C_{0}}{g} v(t)
$$

where $V_{D C}$ is the applied DC bias, $g$ the gap between plates, $A$ the plate area, and $C_{0}=\varepsilon_{0} A / g$ is the capacitance between the plates. Consequently $\left\langle|I(\omega)|^{2}\right\rangle=\left(V_{D C} C_{0} / g\right)^{2}\left\langle|V(\omega)|^{2}\right\rangle$ and integrating over the amplifier bandwidth we have for the RMS noise current

$$
i_{R M S}^{2}=\frac{1}{2 \pi}\left(\frac{V_{D C} C_{0}}{g}\right)^{2} \int_{0}^{\omega_{H}} \frac{4 k T \omega_{0} /(m Q)}{\left(\omega-\frac{\omega_{0}^{2}}{\omega}\right)^{2}+\left(\frac{\omega_{0}}{Q}\right)^{2}} d \omega
$$

The argument of the integral in (8) is strongly peaked near the resonant frequency $\omega_{0}$; provided the amplifier bandwidth $\omega_{H}$ encloses the resonant peak the upper limit can be extended to infinity. In this case the result is almost independent of $Q$.

Table II shows the predicted noise voltage at the output of the amplifier for an amplifier gain of $100 \mathrm{~V} / \mathrm{V}$ and in input resistor of $160 \mathrm{k} \Omega$. The RMS noise voltage is independent of the $Q$ and as all our devices have the same area and gap the only parameter which can lead to different noise voltage is the DC bias ( $9 \mathrm{~V}$ for all transducers). Also shown is the measured RMS noise voltage for three transducers with an amplifier of bandwidth $800 \mathrm{kHz}$. The measured and predicted noise voltages are roughly equal, suggesting that the amplifier noise and interference do not limit performance of the transducer.

Table II. Predicted and measured RMS noise at output of amplifier.

\begin{tabular}{|c|c|c|}
\hline $\begin{array}{c}\text { Frequency } \\
{[\mathbf{k H z}]}\end{array}$ & $\begin{array}{c}\text { Noise Predicted } \\
{[\mathbf{m V}]}\end{array}$ & $\begin{array}{c}\text { Noise Measured } \\
{[\mathbf{m V}]}\end{array}$ \\
\hline 126 & 12 & 14 \\
\hline 255 & 12 & -- \\
\hline 351 & 12 & 22 \\
\hline 404 & 12 & -- \\
\hline 500 & 12 & 19 \\
\hline
\end{tabular}

\section{VACUUM SEALING}

As the transducer noise limits the minimum detectable acoustic signal, we now consider factors which can further improve the transducer performance. Both detected signal and transducer noise scale in the same way with area and electrode gap, so these parameters cannot be used to improve performance. However the signal is linearly dependent on $Q$ and the noise is independent of $Q$. Consequently the signal-to-noise ratio is improved in transducers with higher $Q$. We have determined that it is impractical to obtain any significant further improvement in $Q$ by increasing the number of etch release holes. As a result we have explored packaging transducers in vacuum as is often done with MEMS accelerometers. While there exist production technologies for sealing packages [8], these are 
difficult to implement at the laboratory scale. Instead we have used lead-tin solder to attach a metal lid with a small hole to the package. Then the package is placed in a vacuum chamber and the small hole is sealed with solder while the package is still under vacuum. Fig. 5 shows the sealed package.

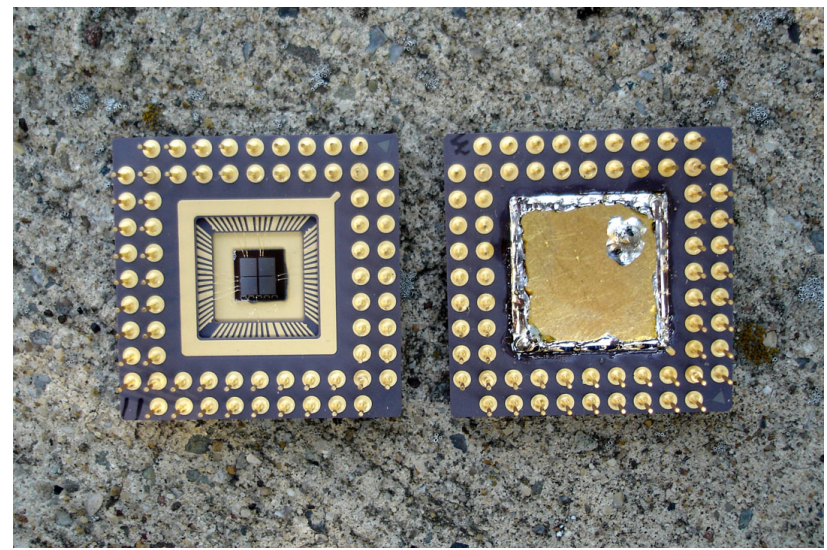

Figure 5. Photograph showing (left) chip attached to package and wirebonded and (right) after evacuation and sealing.

Fig. 3 also shows the admittance magnitude for a transducer in a vacuum-sealed package. The $Q$ is substantially increased; extracted values are presented in Table I. The measured admittance is unchanged after one month or more, indicating that the leak rate is very low.

\section{SUMMARY}

We have reported on the characterization of MEMS acoustic emission transducers. When closely integrated with a well-designed amplifier, the performance of these transducers is limited by Brownian noise. The measured noise magnitude is in good agreement with the predictions of theory, which indicate that noise is independent of the $Q$ the transducer. Finally, we have described a simple method for sealing the transducers in vacuum which is expected to substantially increase the signal to noise ratio.

\section{ACKNOWLEDGMENT}

We thank the Pennsylvania Infrastructure Technology Alliance and the National Science Foundation for support. This material is based upon work supported by the National Science Foundation under Grant No. CMS-0329880. Any opinions, findings, and conclusions or recommendations expressed in this material are those of the authors and do not necessarily reflect the views of the National Science Foundation.

\section{REFERENCES}

[1] D. Ozevin, D.W. Greve, I.J. Oppenheim, and S.P. Pessiki, "Resonant capacitive MEMS acoustic emission transducers," Smart Mater. Struct. vol. 15, pp. 1863-1871, November, 2006.

[2] MEMSCAP, Inc., Durham, NC, http://www.memsrus.com/ncmumps.html.

[3] D.W. Greve, W. Wu, and I.J. Oppenheim, "Modes and Damping in cMUT Transducers for Acoustic Emission," 2006 IEEE Ultrasonics Symposium, pp. 661-664, October, 2006.

[4] Package type CPG06856 (Spectrum Semiconductor Materials, Inc., San Jose, CA)

[5] BIPAX TRA-DUCT 2958 silver epoxy, Tra-Con Inc., Bedford, MA.

[6] D. Ozevin, S.P. Pessiki, D.W. Greve, I.J. Oppenheim, "Adapting a cMUT transducer to detect acoustic emissions," 2005 IEEE Ultrasonics Symposium, pp. 956-959, September, 2005

[7] T.B. Gabrielson. "Mechanical-thermal noise in micromachined acoustic and vibration sensors," IEEE Trans, Electron. Devices, Vol. 40, pp. 903-909, May 1993.

[8] P.K. Khanna, S.K. Bhatnagar, and W. Gust, "Analysis of packaging and sealing techniques for microelectronic modules and recent advances," Microelectronics International vol. 16/2, pp. 8-12, 1999. 